though it could be a necessary correction for techniques employing different geometries. The procedure represented by the application of Eq. (7) corrects for the combined effect of the container wall and the holes in the cavity.

\section{Plasma density measurements}

The motivation for the present work was the requirement for accurate electron densities in the positive column of a glow discharge in helium over a range of discharge currents and gas pressures. ${ }^{8}$ Using a quartz tube with $0.4-\mathrm{mm}$-thick walls, the resonance frequency shifts $\Delta f_{d}$ were first determined for the six dielectric slugs described above. The quartz tube was then made part of the positive column of the helium discharge and the frequency shifts $\Delta f_{p}$ were then observed for the desired set of pressures and currents. The integrals $g_{d}$ and $g_{p}$ of Eq. (7) were machine calculated, now using for convenience the theoretical distributions $E_{0}^{2}(r)$ of Eq. (8). Of the set of values of $f_{d}$, the one chosen in a particular density determination made the ratio $\Delta f_{d} / \Delta f_{p}$ nearest unity. Equation (7) then yielded the corresponding electron density. The densities obtained were about $16 \%$ larger than would have been obtained without the refined calibration procedure described above.

\section{Microwave bridge measurement}

Frequently, plasma densities are measured in a microwave bridge circuit ${ }^{4}$ rather than by a frequency shift of a resonator, and the field attenuation and distortions as introduced by the dielectric container walls (plus that of the holes in the waveguides, or of the distorted an- tenna radiation fields) are sources of errors of the same magnitude as in the above case. For example, it was found that a 1-mm-thick Pyrex tube (same dimensions as above) inserted in an $X$-band waveguide reduced the measured phase shifts of the dielectric samples by $74 \%$ ! A quartz tube of the same wall thickness introduced similarly $32 \%$ error. Such errors can be avoided by the simple calibration procedure outlined above.

\section{CONCLUSION}

It has been shown that plasma containers made from quartz or Pyrex will attenuate microwave fields appreciably and that holes cut into resonance cavities will distort fields, with each effect leading to significant errors in microwave density measurements. However, when proper calibration procedures are employed, such errors can be minimized. It is thus recommended that the calibration procedure described above be followed when large errors in electron density measurements are to be avoided.

*Work supported by the Joint Services Electronics Program, under Contract No. F44620-71-C-0091.

†Present address: Department of Chemistry, Indiana University, Bloomington, Ind. 47405

${ }^{1}$ M. A. Biondi, Rev. Sci. Instrum. 22, 500 (1951)

${ }^{2}$ S. C. Brown and D. J. Rose, J. Appl. Phys. 23, 711 (1952).

${ }^{3}$ J. C. Ingrahm and S. C. Brown, The Applications of Plasmas to Chemical

Processing (MIT, Cambridge, Mass., 1967), Chap. 5.

${ }^{4}$ J. C. Slater, Rev. Mod. Phys. 18, 481 (1946).

${ }^{5}$ We are grateful to Emerson and Cuming, Inc., Dielectric Materials Division, Canton, Mass.

${ }^{6}$ N. Marcuvitz, Waveguide Handbook, MIT Radiation Laboratory Series

(McGraw-Hill, New York, 1951).

${ }^{7}$ M. Sicha, G. G. Cloutier, and R. Bolton, Can. J. Phys. 45, 3979 (1967)

${ }^{8}$ H. F. Wellenstein and W. W. Robertson, J. Chem. Phys. 56, 1072 (1972); J.

Chem. Phys. 56, 1077 (1972); J. Chem. Phys. 56, 1411 (1972)

\title{
Cerenkov and transition radiation in space-time periodic media
}

\author{
Charles Elachi \\ Jet Propulsion Laboratory and California Institute of Technology, Pasadena, California 91109 \\ (Received 17 March 1972) \\ The solution to the problem of determining the radiation emitted by a uniformly moving charged particle in a \\ sinusoidally space-time periodic medium is obtained. The space-time periodicity can be considered as due to a strong \\ pump wave and is expressed as a traveling-wave-type change in the dielectric constant or the plasma density. The \\ solution covers also the limiting case of sinusoidally stratified media. The expression and spectrum of the radiated \\ electromagnetic field are determined for different media: dielectric, isotropic and uniaxial plasma. Depending on the \\ nature of the medium and the velocity of the particle, the radiated field is of the Cerenkov and/or transition type. The \\ Brillouin diagram is used extensively in understanding and determining the nature, extent, and spectrum of the different \\ modes of radiation, and a focusing effect is also studied.
}

\section{INTRODUCTION}

When a charged particle moves with a constant speed in a medium, there are two types of radiation, Cerenkov and transition, which may be emitted depending on the velocity of the particle and the nature of the medium. Cerenkov radiation is emitted when the particle velocity is higher than the phase velocity of light in a dielectric, ${ }^{1,2}$ and in a magnetoplasma. ${ }^{3}$ Transition radiation is emitted when the medium is inhomogeneous. ${ }^{4-6}$

In this paper we determine the radiation field of a charged particle moving uniformly in the $z$ direction (Fig. 1) in a generally space-time periodic mediumi.e., dielectric constant or plasma density behave like $\eta(z, t)=\eta_{0}\left[1+\eta_{1} f(K z-\Omega t)\right]$, where $f(\xi)$ is a periodic function. The space-time periodicity can be considered as due to a strong pump wave. Such a case can be encountered in devices, in the earth ionosphere submitted to high-power radar pulses, ${ }^{7}$ planetary ionosphere disturbed by the motion of a large body (like the satellite Io moving in the Jovian atmosphere and the resulting correlation with the Jovian decametric emission), and others.

Once the field is determined, our objective is to show that by using the Brillouin diagram for the limit $\eta_{1} \rightarrow 0$, the nature and the spectrum of the radiated field, diagram discontinuities, and focusing effects can be determined and studied using elementary computations. 


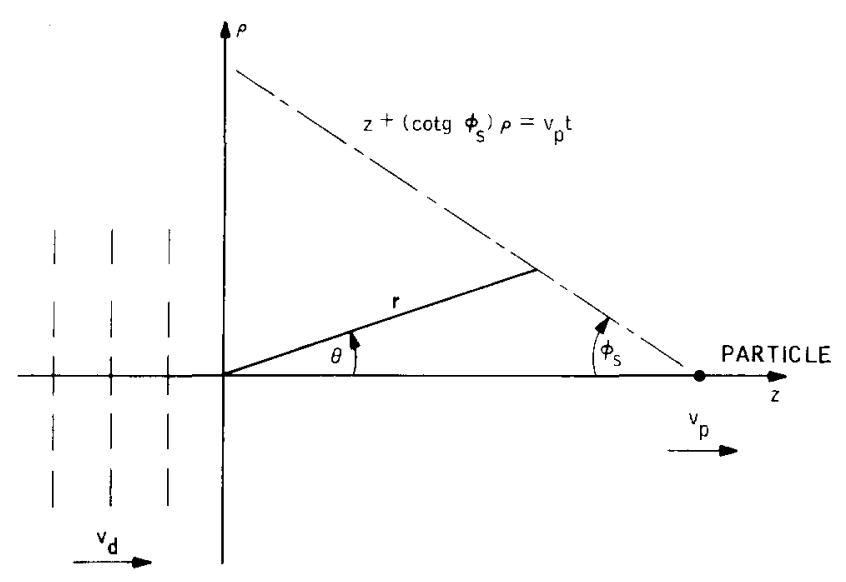

FIG. 1. Particle uniformly moving in a space-time periodic medium (geometry).

\section{GENERAL SOLUTION}

Let us consider a charged particle moving with constant velocity in a medium modulated in a wavelike manner:

$$
\eta(z, t)=\eta_{0}\left[1+\eta_{1} f(K z-\Omega t)\right]
$$

where $\eta(z, t)$ is the dielectric constant $\epsilon$ or plasma density $N$ depending on the medium considered; $f(\xi)$, the periodic function, is equal to $\sum_{m=-\infty}^{m=+\infty} a_{m} \exp (i m \xi) ; \Omega$ and $K$ are the angular frequency and wave number, respectively, of the disturbance.

The radiated field, if it exists, is of the TM type relative to the $z$ axis (i.e., $\mathrm{H}=H \mathrm{e}_{\phi}, \mathrm{E}=E_{\rho} \mathrm{e}_{p}+E_{z} \mathrm{e}_{z}$ ). In the case of a plasma or uniaxial plasma (static field $B_{0}$ $=B_{0} \mathbf{e}_{z}$ with $B_{0}$ large) we also use the vector $\mathbf{A}=\partial \mathbf{E} / \partial t$. This field consists of an infinite set of harmonics (due to the time periodicity) and modes (due to the space periodicity). Therefore, using the separation of variables method and Floquet's theorem, we can write an elementary solution for the field:

$$
\begin{aligned}
H^{(e)}= & \sum_{n=-\infty}^{n=+\infty} \sum_{q}^{q=-\infty} H_{n q} H_{1}^{(1)}\left(\delta_{q} \rho\right) \exp [i(\kappa+n K) z-i(\omega+n \Omega) t], \\
\mathrm{V}^{(e)}= & \sum_{n=-\infty}^{n=+\infty} \sum_{q=-\infty}^{q=+\infty}\left[-i V_{n q q} H_{0}^{(1)}\left(\delta_{q} \rho\right) \mathbf{e}_{z}+V_{n q \rho} H_{1}^{(1)}\left(\delta_{q} \rho\right) \mathbf{e}_{\rho}\right] \\
& \times \exp [i(\kappa+n K) z-(\omega+n \Omega) t]
\end{aligned}
$$

where $\mathbf{V}=\mathbf{E}$ or $\mathbf{A}$, depending on whether the medium is a dielectric or a plasma; $H_{n q}, V_{n q z}$, and $V_{n q \rho}$ are the amplitude of the $n$th harmonic and $q$ th mode; $\omega, \kappa$, and $\delta_{a}$ are separation variables. (The index $q$ corresponds to the different solutions of the dispersion equation as we will see later.); $H_{0}^{(1)}$ and $H_{1}^{(1)}$ correspond to the Hankel function.

Putting the expressions of $\mathrm{V}^{(e)}$ and $H^{(e)}$ in Maxwell's and the linearized force equations, replacing $\epsilon$ or $N$ by its Fourier series, and equating the terms with identical $z$ and $t$ dependence, we find the following infinite systems of linear equations ${ }^{8-10}$ (the index $q$ is disregarded for the moment):
For a dielectric or a plasma,

$$
\begin{aligned}
& D_{n} V_{n x}+\sum_{l=-\infty}^{l=+\infty} a_{n-l} V_{l x}+G_{n} V_{n z}=0, \\
& D_{n}^{\prime} V_{n z}+\sum_{l=-\infty} a_{n-t} V_{l z}+G_{n} V_{n x}=0,
\end{aligned}
$$

for a uniaxial plasma,

$$
D_{n} V_{n z}+\sum_{i=-\infty}^{t=+\infty} a_{n-l} V_{l z}=0
$$

where $D_{n}, D_{n}^{\prime}$, and $G_{n}$ are given in Table I. These systems can be written in a matrix form

$$
\|M\| \cdot\left|V_{z}\right|=0
$$

where $\left|V_{z}\right|$ is an infinite column vector of elements $V_{n z}$ and $\|M\|$ is an infinite matrix whose elements can be determined from Eqs. (3) or (4).

The nontriviality condition of the above system gives the dispersion relation

$$
\Delta(\delta, \kappa, \omega)=\operatorname{det}|| M||=0 .
$$

For a fixed $\omega$, we get $\delta=g(\kappa)$ which gives the wave vector diagram. ${ }^{7}$ For each value of $k$ corresponds many solutions $\delta=\delta_{a}$, where $q=0, \pm 1, \ldots$ represents the mode.

The relative amplitudes $C_{n q}^{\prime}=V_{n q g} / V_{0 q z}, F_{n q}^{\prime}=V_{n q x} / V_{0 q z}$, and $A_{n q}^{\prime}=H_{n q} / V_{0_{q}}$ can be determined from Eq. (5) and Maxwell's equations. The reference values $V_{0 q \varepsilon}$ will be determined from the source condition as we will see later.

The complete expression of the field is obtained by integrating the elementary solution over all the values of
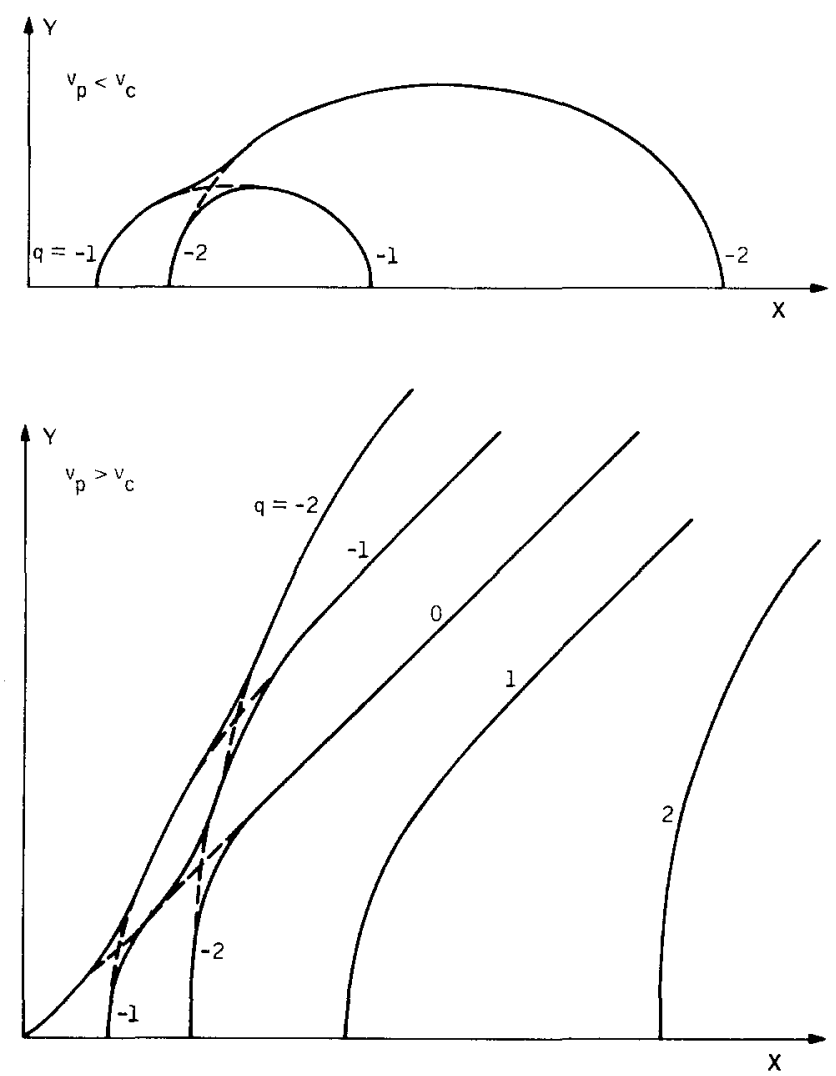

FIG. 2. Brillouin diagram for a dielectric $\left(v_{p} \geq v_{c}\right)$ or a plasma $\left(v_{p}<v_{c}\right)$. 
TABLE I. Parameters. $\left[\kappa_{n}=\kappa+n K, \beta_{n}=(\omega+n \Omega) / v_{c}, \beta_{p 0}=\omega_{p 0} / v_{c}\right.$, and $\omega_{p 0}$ is the plasma frequency when $\left.\eta_{1}=0.\right]$

\begin{tabular}{lllc}
\hline & \multicolumn{1}{c}{$\eta_{1} D_{n}$} & $\eta_{1} D_{n}^{\prime}$ & $\eta_{1} G_{n}$ \\
\hline Dielectric & $1-\left(\kappa_{n} / \beta_{n}\right)^{2}$ & $1-\left(\delta / \beta_{n}\right)^{2}$ & $\delta \kappa_{n} / \beta_{n}^{2}$ \\
Plasma & $1-\left(\beta_{n}^{2}-\kappa_{n}^{2}\right) / \beta_{p 0}^{2}$ & $1-\left(\beta_{n}^{2}-\delta^{2}\right) / \beta_{p 0}^{2}$ & $\delta \kappa_{n} / \beta_{p 0}^{2}$ \\
Uniaxial plasma & $1-\left(\beta_{n} / \beta_{p 0}\right)^{2}\left[1-\delta^{2} /\left(\beta_{n}^{2}-\kappa_{n}^{2}\right)\right]$ & & \\
\hline
\end{tabular}

$\kappa$ and $\omega$ using an adequate weighting function. But as we will see later, $\omega$ is uniquely related to $\kappa$ and a simple integration over $\omega$ is sufficient. Therefore we can write

$$
\begin{aligned}
\mathrm{H}= & \mathbf{e}_{\phi} \sum_{n} \sum_{q} \int_{-\infty}^{+\infty} W_{q}(\omega) H_{n q} H_{1}^{(1)}\left(\delta_{q} \rho\right) \\
& \times \exp [i(\kappa+n K) z-i(\omega+n \Omega) t] d \omega .
\end{aligned}
$$

The set of weighting functions $W_{q}$ is determined at the same time as $V_{0_{q z}}$ from the source condition

$$
\lim _{\rho \rightarrow 0} 2 \pi \rho H=e v_{\rho} \delta\left(z-v_{p} t\right),
$$

where $\delta(\xi)$ is the $\delta$ function. Taking $\alpha_{q}=W_{q} V_{0 q z} / \delta_{q}$, writing $\delta\left(z-v_{p} t\right)$ in an integral form, and replacing $H$ by its expression from Eq. (6) we find

$$
\begin{aligned}
& \int_{-\infty}^{+\infty}\left\{\sum_{n} \sum_{\alpha} \alpha_{a} A_{n q}^{\prime} \exp [i(\kappa+n K) z-i n \Omega t]\right. \\
& \left.-\frac{1}{4} i e \exp \left[\left(i \omega / v_{\rho}\right) z\right]\right\} \exp (-i \omega t) d \omega=0 .
\end{aligned}
$$

This relation must be satisfied for all values of $z$ and $t$, therefore we must have

$$
\begin{aligned}
& \kappa=\omega / v_{p}, \\
& \sum_{q} \alpha_{q} A_{n q}^{\prime}=0, \quad \text { for } n \neq 0 \\
& \sum_{q} \alpha_{q} A_{0 q}^{\prime}=\frac{1}{4} i e .
\end{aligned}
$$

The two last relations can be written in a matrix form

$$
\left.\left\|A^{\prime}|| \cdot|\alpha|=|P| \Rightarrow|\alpha|=\right\| A^{\prime}||\right|^{-1} \cdot|P|,
$$

where $\left\|A^{\prime}\right\|$ is an infinite matrix with elements $A_{n q}^{\prime}$ which are known, $|\alpha|$ is an infinite column vector with elements $\alpha_{a}$, and $|P|$ is an infinite column with elements zero except the central one which is equal to $\frac{1}{4} i e$.

Equation (7) determines all the elements $\alpha_{q}$ and the field is given by

$$
\begin{aligned}
\mathbf{H}= & \mathbf{e}_{\phi} \sum_{n} \sum_{q} \int_{-\infty}^{+\infty} \alpha_{q} \delta_{q} A_{n q}^{\prime} H_{1}^{(1)}\left(\delta_{q} \rho\right) \\
& \times \exp \left[i\left(\omega / v_{p}+n K\right) z-i(\omega+n \Omega) t\right] d \omega
\end{aligned}
$$

and $\delta_{q}(\omega)$ is determined from the dispersion equation where $\kappa$ is replaced by $\omega / v_{p}$.

\section{FAR FIELD}

Using the steepest descent method, the integral in Eq. (8) can be determined for $r=\rho / \sin \theta \rightarrow \infty$ and we get

$$
\begin{aligned}
H= & -\frac{2 i}{r \sin \theta} \sum_{q} \sum_{n} \sum_{s} \alpha_{q s} A_{\text {rqs }}^{\prime}\left(\frac{\delta_{q s}}{\left|\delta_{q s}^{\prime \prime}\right|}\right)^{1 / 2} \\
& \times \exp \left[i \chi_{q s} r+\frac{1}{4} i \pi\left(1-\operatorname{sign} \delta_{q s}^{\prime \prime}\right)\right],
\end{aligned}
$$

where $\delta_{q}^{\prime \prime}=d^{2} \delta_{q} / d \omega^{2}, \chi_{q}=\delta_{q} \sin \theta+\left(\omega / v_{p}+n K\right) \cos \theta-(\omega$ $+n \Omega) t / r$, and the index $s$ means the value at the saddle points $\omega=\omega_{s}$ which are determined by

$$
\left.\frac{d \chi_{a}}{d \omega}\right|_{\omega=\omega_{s}}=\left.0 \Rightarrow \sin \theta \frac{d \delta_{q}}{d \omega}\right|_{\omega=\omega_{s}}+\frac{\cos \theta}{v_{b}}-\frac{t}{r}=0
$$

which can be written as (see Fig. 1)

$$
Z+v_{p} \delta_{q}^{\prime}\left(\omega_{s}\right) \rho=v_{p} t
$$

where $\delta_{q}^{\prime}\left(\omega_{s}\right)=d \delta_{q} /\left.d \omega\right|_{\omega=\omega_{s}}$. This represents a cone of axis $z$, vertex at $v_{p} t$ (which is the position of the particle) and half-angle

$$
\phi_{Q s}=\operatorname{arccotg}\left[v_{p} \delta_{Q}^{\prime}\left(\omega_{s}\right)\right] .
$$

Hence, for a given mode of radiation $q$, the band of the spectrum around $\omega_{s}$ (and $\omega_{s}+n \Omega$ ) will be concentrated, in space, on the surface of a cone attached to the parti cle, with axis parallel to $\mathrm{e}_{z}$ and half-angle $\phi_{q s}$.

\section{BRILLOUIN DIAGRAM AND SPECTRUM}

The exact solution of the dispersion equation requires a large amount of numerical computation. Another approach is to take the limit as $\eta_{1} \rightarrow 0$ which can be studied analytically and graphically and gives accurate information on the nature and spectrum of the radiated field, the diagram discontinuities, and the focusing effect.

At the limit $\eta_{1} \rightarrow 0$, the dispersion equation reduces to

$$
\begin{array}{ll}
D_{q} D_{q}^{\prime}=G_{q}^{2} & \text { for a dielectric or plasma, } \\
\eta_{1} D_{q}=0 & \text { for a uniaxial plasma. }
\end{array}
$$

Replacing $D_{q}, D_{q}^{\prime}$, and $G_{q}^{2}$ by their expressions from Table I we obtain the following:

for a dielectric, $\quad Y^{2}+\left(1-R^{2}\right)(X+q L)^{2}=(q B)^{2}$;

for a plasma, $\quad Y^{2}+\left(1-R^{2}\right)(X+q L)^{2}=(q B)^{2}-a^{2}$;

for a uniaxial plasma,

$$
Y^{2}=\left[(X+q)^{2}-\left(R X+q R^{\prime}\right)^{2}\right]\left[-1+a^{2} /\left(R X+q R^{\prime}\right)^{2}\right] .
$$

The parameters used are given in Table II. In the rest of the study we assume $R^{\prime}<1$ (i.e., $v_{d}<v_{c}$ ).

\section{A. Isotropic dielectric and plasma}

For $R<1$ (which is always the case in a plasma), the Brillouin diagram consists of a family of ellipses and only the modes $q<0$ (for a dielectric) and $q<-|a / B|$

TABLE II. Notations.

\begin{tabular}{ll}
\hline$Y=\delta / K, \quad X=\omega / K v_{p}$ \\
$v_{c}=\left(\mu_{0} \epsilon_{0} \epsilon_{r^{\prime}}\right)^{-1 / 2}$ \\
$v_{d}=\Omega / K=$ velocity of disturbance \\
$R=v_{p} / v_{c}, \quad R^{\prime}=v_{d} / v_{c}$ \\
$L=\left(1-R R^{\prime}\right) /\left(1-R^{2}\right)$ \\
$B^{2}=\left(R-R^{\prime}\right)^{2} /\left(1-R^{2}\right)$ \\
$a=\omega_{p 0} / K v_{c}$
\end{tabular}



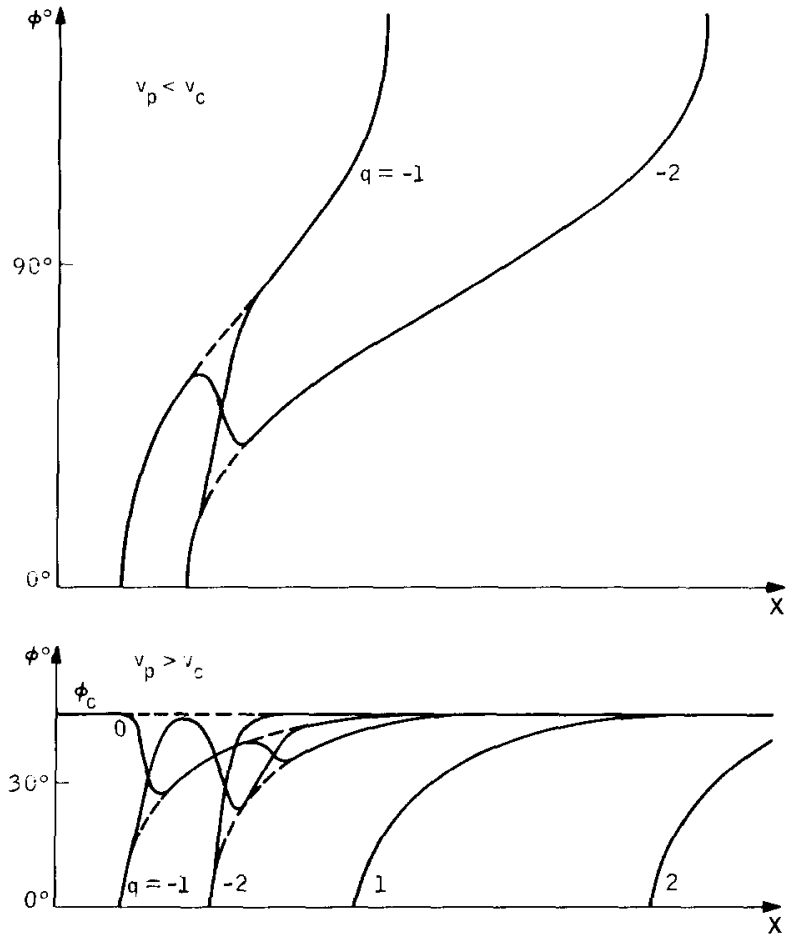

FIG. 3. Cone angle $\phi$ function of $X=\omega / K v_{p}$.

(for a plasma) radiate a transition-type radiation. In Fig. 2 we present the first two modes $(q=-1,-2$ for a dielectric). The spectrum of each mode consists of a series of bands given by (value of $X$ for $Y=0$ )

$$
m_{q}+n \Omega<\omega_{n q}<M_{q}+n \Omega,
$$

where $m_{q}$ and $M_{q}$ are, respectively, the smaller and larger of the two roots: $K v_{p}\left[-q L \pm\left(q^{2} B^{2}-a^{2}\right)^{1 / 2} /\left(1-R^{2}\right)\right]$. For the dielectric we have $a=0$. For a certain band of the spectrum, the higher part corresponds to $\phi=180^{\circ}$ and the lower part to $\phi_{s}=0^{\circ}$ (see Fig. 3).

The intersection point between two modes $q$ and $q^{\prime}$ can be easily determined as

$$
X_{a q^{\prime}}=-\frac{q+q^{\prime}}{2}\left(\frac{1-R^{\prime 2}}{1-R R^{\prime}}\right) .
$$

All the above results are independent of the function $f(\xi)$.

For $\eta \neq 0$, strong interaction occurs at the intersection points between different modes leading to discontinuities in the diagram (see Fig. 4). The spectrum bands and the location of the discontinuities can be still well approximated by Eqs. (17) and (18). This was verified numerically and it agrees perfectly with the studies done by other authors ${ }^{5,6}$ for the stratified case $\left(v_{d}=0\right)$. In this special case, we have $X_{q q^{\circ}}=\frac{1}{2}\left(q+q^{\prime}\right)$ which is independent of $R$. The function $f(\xi)$ has major importance if the accurate behavior near the interaction regions is required.

In the case of a dielectric, the above results are not valid for $v_{d} \simeq v_{c}$ which correspond to the sonic region. ${ }^{11}$
For $R>1$ (in a dielectric), the Brillouin diagram is a family of hyperbolas for $q \neq 0$ and a straight line for $q$ $=0$. The spectrum's bands are given by (see Fig. 2)

$$
\begin{aligned}
& \omega_{n q} \geq-q K v_{p} \frac{1-R^{\prime}}{1-R}+n \Omega \text { for } q \geq 0 \\
& \omega_{n q} \geq-q K v_{p} \frac{1+R^{\prime}}{1+R}+n \Omega \text { for } q \leq 0 .
\end{aligned}
$$

The lower part of a band is radiated at $\phi_{s}=0^{\circ}$ and the higher part at $\phi_{s}=\phi_{c}=\arcsin (1 / R)$. The mode $q=0$ is radiated at $\phi_{s}=\phi_{c}$ which is the Cerenkov angle in the homogeneous case. For $\eta_{1} \neq 0$, there are discontinuities at the points given by Eq. (18), and the angular distribution of the field changes near these discontinuities but always $\phi_{s} \leq \phi_{c}$ (Figs. 3 and 4 ).

\section{B. Stratified uniaxial plasma}

Taking the limit $v_{d}=0$, the Brillouin diagram in the limit $\eta_{1} \rightarrow 0$ is given by

$$
Y^{2}=\left(R^{2} X^{2}-a^{2}\right)\left\{1-[(X+q) / R X]^{2}\right\}
$$

and the spectrum is given by

$$
\begin{array}{ll}
\omega \leq \omega_{p 0} & \text { for } q \geq 0 \\
\omega \leq S \text { and } M \leq \omega \leq N & \text { for } q<0,
\end{array}
$$

where $S, M$, and $N$ are, respectively, the smallest, inbetween, and largest of the three terms:
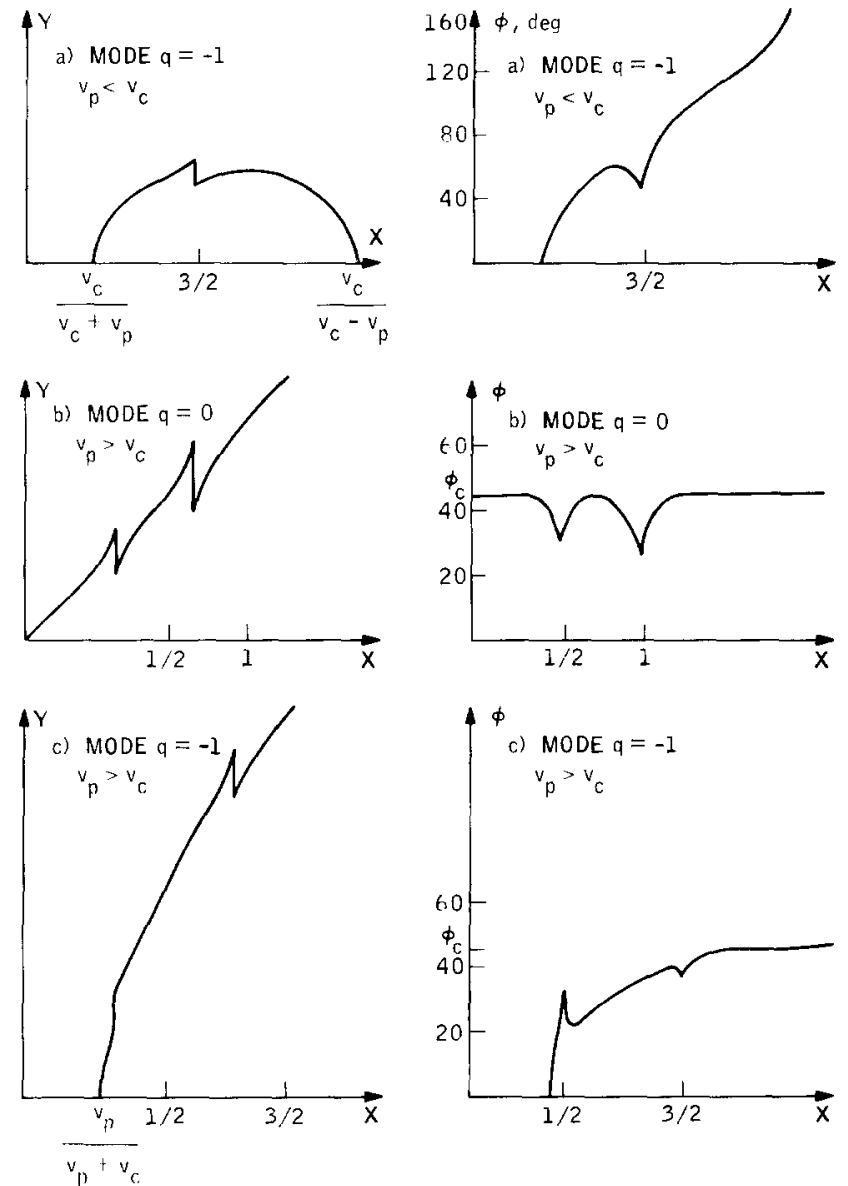

FIG. 4, Brillouin diagram and cone angle for different modes $\left(\eta_{1} \neq 0\right)$ when $v_{d}=0$ 

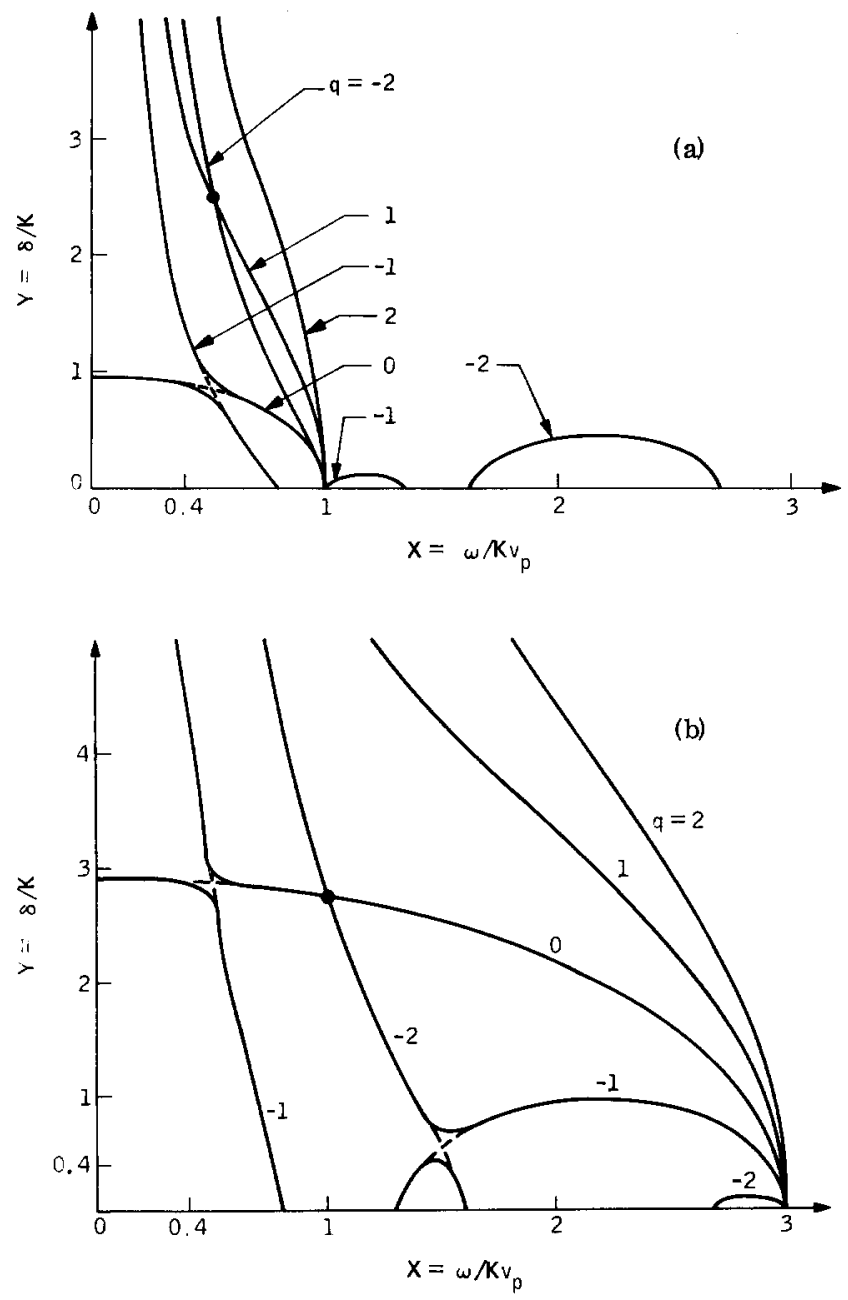

FIG. 5. Brillouin diagram in a uniaxial plasma, (a) $R=0.25$ and $a=0.25$; (b) $R=0.25$ and $a=0.75$.

$$
\omega_{p 0}, \quad|q| K v_{p} /(1+R), \text { and }|q| K v_{p} /(1-R) .
$$

In Fig. 5 we present two Brillouin diagrams. The behavior near the intersection points of two successive modes was determined using a first-order Taylor series development and verified numerically for the case of a sinusoidal stratification with only the two successive modes taken into account.

\section{FOCUSING EFFECT}

For $\eta_{1} \neq 0$, the Brillouin diagrams studied above have inflection points where $\delta_{q}^{\prime \prime}(\omega)=0$. This leads to a large field at the corresponding frequency and angle [Eq. (9)]. This is due to the focusing in the inhomogeneous medium. ${ }^{9}$ This effect can be of interest in studying the transition radiation in a plasma where no Cerenkov cone exists.

\section{CONCLUSION}

The formal solution for the radiation field of a uniformly moving charged particle in a space-time periodic medium has been obtained. We showed that the Brillouin diagram in the limit $\eta_{I} \rightarrow 0$ is very helpful in determining with good approximation the frequency and angular spectrum, and the discontinuities which occur when $\eta_{1} \neq 0$ without any involved numerical computation. We also pointed out the focusing effect caused by the inhomogeneity. This effect may be of interest in determining the properties of the medium or the velocity of the particle, especially in the case of the plasma where there is no Cerenkov cone.

\section{ACKNOWLEDGMENTS}

The author deeply thanks Professor C.H. Papas for many helpful discussions during this study. This paper presents the results of one phase of research carried out at the Jet Propulsion Laboratory, California Institute of Technology, under Contract No. NAS7-100 sponsored by the National Aeronautics and Space Administration, and at the California Institute of Technology, under Air Force Grant AFOSR-68-1400.

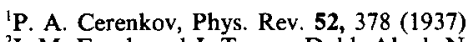

${ }^{2}$ I. M. Frank and I. Tamm, Dokl. Akad. Nauk SSSR 14, 109 (1937)

${ }^{3} \mathrm{~J}$. V. Jelley, Cerenkov Radiation and Its Applications (Pergamon. New York, 1958)

${ }^{4} \mathrm{~K}$. Casey, Can. J. Phys. 46, 1957 (1968).

${ }_{5}^{5}$ K. F. Casey, C. Yeh, and Z. A. Kaprielian, Phys. Rev. B 40, 768 (1965).

${ }^{6}$ K. F. Casey and C. Yeh, Phys. Rev. A 2, $810(1970)$.
${ }^{7}$ W. F. Utlaut and R. Cohen, Science 174, 245 (1971).

${ }^{8}$ C. Elachi, IEEE Trans. Antennas Propag. 20, 534 (1972).

${ }^{9}$ C. Elachi, IEEE Trans. Antennas Propag. 20, 534 (1972).
${ }^{\circ}$ C. Elachi, IEEE Trans. Antennas Propag. 20, 280 (1972).

${ }^{10} \mathrm{C}$. Elachi, Ph.D. thesis (California Institute of Technology, 1971)

(unpublished)

${ }^{11}$ E. S. Cassedy and A. A. Oliner, Proc. IEEE 51, 1342 (1963). 